\title{
Reconfigurable of Machine Chain for Support of Innovation
}

\section{Štefan Valenčík ${ }^{1, *}$}

${ }^{1}$ Technical University of Košice, Faculty of Mechanical Engineering, Department of Production Technigues, Letná 9, 04000 Košice, Slovakia

\begin{abstract}
The paper presents the base of knowledge from the field of architecture and development of reconfigurable machine system of producing and operating technique, including their formation to specific machine system in the accordance with strategy of development. The core of paper makes new approaches solutions, which results in the technical new conceptions of automatic structures, which are able to work in specific place. Solution is standing primarily on more variant synthesis constructive element, which allows obtain untraditionally textures susceptible not only concentration motion intro- individual working knot (tool and, objective), but also concentration functions and running intro-general configuration machine system.
\end{abstract}

Keywords: Producing technique, operating technique, dynamic module, integrated kinematics.

\section{Introduction}

A new approach to the management of production (lean, agile), rapid adaptation in response to market demands, which dates from 90-years [14] require a focus on the next generation of manufacturing and handling equipment using reconfiguration ideas in two directions basically:

- combining of maximum number of different technology/handling operations in a single unit [1],

- developing of reconfigurable machines [4].

A new generation of production and handling machines capable of reconfiguration of their structure in order to adjust the machine to the requirements of the production task require a new design approach on systems and machine level also. The key core moments by these approaches are connected with:

- development of basic principles and methods for design and analysis of reconfigurable machine and production systems,

- design and development of Reconfigurable Machine Tools (RMT), responsible of the key characteristics for reconfiguration (modularity, scalability, inerrability, convertibility, diagnostic ability and customization).

We are talking about new generation of products known as adaptive products, which are able to solve the adaptability for individual phases of the life cycle (phase of development, production and / or utilization), that extends and expands their functionality over time. Traditional NC machines and new generation of CNC machines equipped multifunctional features are examples of this. While the traditional ones, solving not only adaptability but also operability processes are not enough today, using a new ones is critical, especially if the new constructionaltechnological elements (materials, energy / transformation / distribution members, IT) directly implemented to CNC machine allow in phase its using to apply progressive forms of operational utilization on the machine (more complicated 
shapes of products, achieving higher performance and higher quality).

It can be concluded from the mentioned that the dynamics of development requires concentration to solve the requirements concerning ability to respond and adapt the changes of state in production. These are transformed to a model of cognitive adaptability, variability, auto diagnostics, auto resistance, paradigms of auto-improvements of environment and the coordinated of development [11]. To clarify higher mentioned facts the development of method for generating the structures of re-configurable machine systems with possibility to concentrate more technological/manipulative operations into one device is presented.

\section{1 Trend}

\section{Trend and Goals Solution}

Current trends oriented on development and exploitation capital asset are ahead into the bargain, that is needed to search solution with new and high effect. This solution are mostly coupled with effort about complex, concentrated solution function in the frame of assembly machine, devices and the system with propagator function building modulated and its integrated stays (assembly). Their applying allows develop new conception machine and furnished with sufficient process parameters, with high effectiveness, low weight, with bigger control range and high process reliability. Contribute to some growth concentrated productions, combination several methods, raisings performance parameters, simplification textures technological place of work and primarily to filling claim to custom prepared mechanical systems with custom limited technical and economical parameters.

Intention of integration and reconfiguration of machine system should be considered as e.g. "increase in modality machine of the chain and entire concatenation features and activities". Application logistic structure and development lets high variability applications this techniques thereby, that being downloadable technological professions and maybe lightly arrange and by the change lightly re-arrange. Deepening the integration of logistics and associated production technology helps manufacturers to shift production of fulfilling the requirements of the market. It is desired mainly in the production phase focusing on the integration of product design with respect to the requirement for greater adaptability and agility, and the orientation of industrial experience in an innovative change in corporate restructuring and strategy systems.

\subsection{Goals}

The objectives of integration and reconfiguration of the machine rather than specific objectives (e.g. increased locomotion modalities module or possibly a reference interconnection module). Objectives must be clear and specific, realistic and stimulating at the same time, internally consistent and quantifiable as possible so as to comply with the mission and objectives of integration of material flow. The material is considered to be achieved in a transparent manner. The aims and objectives may not always comply with the technical and implementation options that sometimes contradict each other and thus produce pressures on resources and review how the system works machine. Therefore, the mission profile and the integration of machine reconfiguration scheme should be based on the logic structure constituting of estimated potential agents of development and action potential production companies, such as:

- developments in the field, advanced technological methods and structures, our compatibility and European legislation,

- specific needs, the company seeks to provide, in accordance with the needs of industry practice for innovative change strategies and corporate restructuring schemes, respecting market adaptability, production efficiency, competitiveness and raising the level of service provided,

- way in which the needs will be provided, modalities machine chain, intermodal hubs using the tool and a subject node.

Analysis of potential aims not only to obtain adequate answers to the questions above, but is intended to reflect the strategic measures to develop an integrated and reconfigurable technology. This achieves consistency in the process of developing and maintaining a strategic relationship between the goals, state of the art-production and application status.

\section{Methodology Solution 3.1 The strategy of integration and reconfiguration of machine system}

In the development of machine system with integrated and reconfigurations effects are based on 
the requirements of applied fields and the technical capabilities of the components that are abstracted into a model - Fig. 1. From this could clarify the influencing factors and functional ties.

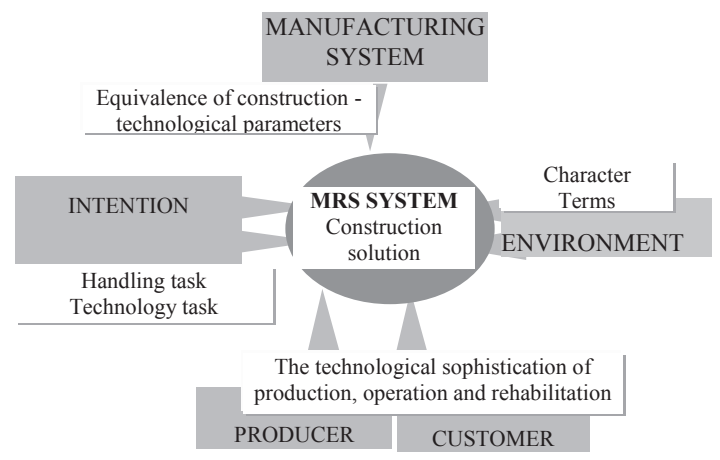

Fig. 1: Factors affecting the structure of the machine system.

The analysis focused on the purpose of handling / confirms the role of technology influencing machine system, in particular the mechanical, physical and topological nature, weight, accuracy and stability of position control method and sorting and object exchange rate of production.

Analysis of the nature and terms of application environment confirms influence machine system, in particular the work area (location, shape, size, input), technological dislocation axis (location, access, function), an interactive relationship systems (function, identifying, blocking, movement forward - backward), energy (distribution, transformation, branching flow), mechanical (unification, separation, variability) and realized the nature of technology / process handling (processing / handling tools, process parameters-load, accuracy, stability and performance time modes).

Analysis of the production system confirms the influence of particular machine based on its technical parameters (power-productivity, positioning accuracy, kinematics and dynamic properties), design parameters (baseline layout for the premises, the building interface), connecting mechanism, the program and energy treatment of cross-links (energy and information interface), and integrated technology-related activities (handling, transport, storage and ancillary).

Analysis of the impact of the manufacturer and the user confirms the influence of particular machine based on the technological possibilities of production, guaranteeing fixed service and maintenance activities, the level of servicing and maintenance, the maintenance of operational capability.

\subsection{Methodology-procedure}

The starting solution is the analysis of stimuli showing the need for greater synergy of elements, nodes and complete and wider application of mechatronics principles. Linked to this is to identify characteristics that affect the composition of the machinery of the system and its impact on the achievement of the objectives of application. The result is a logic machine patterns between the system and the area of application in the form of the model. The model represents a starting base profiling machine systems, which represents our logistics development. The aim is to develop logistics ongoing, linked sequences of individual machine modules (reference, connection, motion), as well as construction machinery reconfiguration availability defined systems and performance parameters.

Based on the above methods and to develop modular analysis of individual machine modules comprising reconfigurable machine system, a case may vary. To improve the user properties are used to address kinematics structure of anomalous elements and their mutual relations, thus creating space for new concepts of plant and equipment of modular organization. Furthermore, the specificities of these concepts mean that they can change the spectrum of tasks, again spread over different modules and used to address new challenges. Moreover, can integrate additional features, e.g.: adding motion to change individual modules and operating system machine work opportunities. Combining machine modules can generate complete machine systems ready for use according to user specification.

\subsection{Construction morphology modular machine system}

Architecture of building modules is based on the needs of the targeted grouping of suitable modules into the architecture of MSS, which is repeatable in the form of structural bases for specific application types. A characteristic feature is that the modules are characterized by high level of conformity (standardization) and autonomy (they can work as stand-alone systems).

This approach allows the formation of products and technology platforms, which leads to effective and flexible production in small production volumes (high variability of the products). Platforms creating is appropriate to realize on the principle of 
grouping suitable modules MS in sets of modular reconfigurable system (MRS), which are repeatable useful in a form of construction base for certain types of applications in the architecture of the MSS. This principle, Fig. 2, has features:

- the platform is set of modules $M S_{i}$ (e.g. $K M_{1}^{m}, I M_{n}^{m}$, $\left.R M_{n}^{m}\right)$ used in more complete sets $M S S_{j}(M P$ - Modules of Platform), e.g. $M S S_{1}, M S S_{2}, \ldots$

- the set of modules $M S_{i}$ is participating in more sets $M S S_{j}$ (MM-Multimachine Modules), e.g. $P_{n m}^{1}$

- the set of modules $M S_{i}$ participating in only one set $M S S_{j}\left(M S\right.$ - Singlemachine Modules), e.g. $P_{n m}^{k}$,

- platform can be extended by complementing other construction modules $M S_{i}$ appropriate to extend the functionality and features of possible options in the set $M S S_{j}$,

- allow assess the level of modularity of created sets through the levels of a constructing modules $M S_{i}$ in a particular set $M S S_{j}$.

Thus adopted platform's concept is understood as a higher form of implementing modular concept of elements of the production system (the minimum number of modules to assemble the largest possible number of variants). It is advisable to assemble structure of the assessed sets the MSS into so-called map of modular system - a summary conception of the structure of individual sets and conception application of individual various variants of constructing modules in sets MSS.

\section{Definition and Principles of the New Solution options}

\subsection{Definition motion module}

For the generation of project reports were received following definition for the motion of the module design and user perspective:

- motion module is compact mechatronic solution. Combines engine, reference, transformation and transmission unit, collection and distribution of energy and member information,

- motion module is a standard or custom product providing the required functions positioning tool / workpiece in one or two NC axes. It is usually the design and technologically compatible with a certain group of modules, building machinery and production systems. In this case, its technical characteristics are closely tied to the final determination of technology-workpiece to which it is built machine system. The movement module may be designed based motion modules with the best accessories from the basic technology destination. This can be achieved with maximum degree of modularity and optimal choice of technical parameters in relation to the price of the product.

\subsection{Principles of development}

Experience to date in addressing the concentration of functions and activities is based on serial links units implementing kinetic functions. Such solutions require increased demands on the spatial arrangement of elements and for the

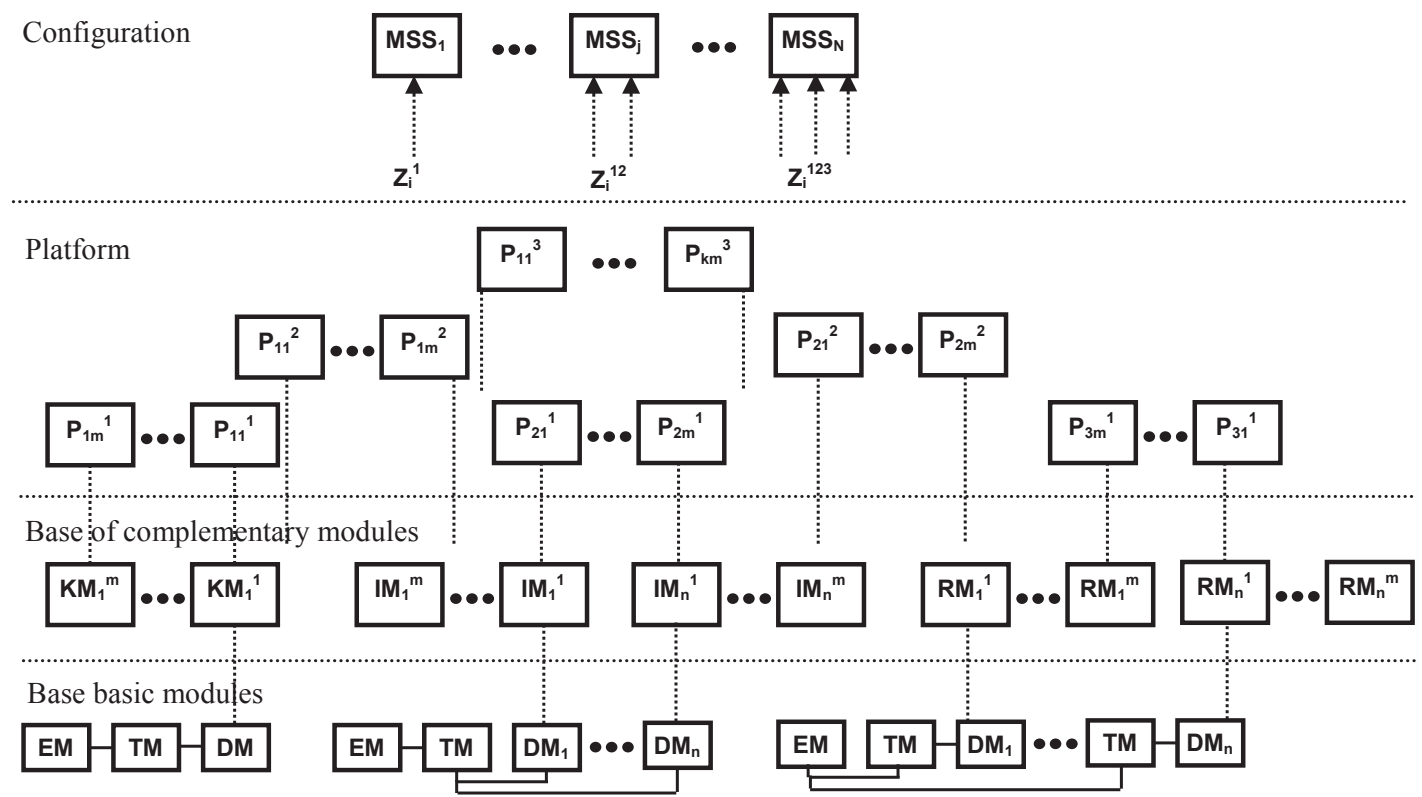

Fig. 2: Construction morphology modular machine system. 
overall concept of kinematics chain, as well as the implementation costs. This encourages the development of new solutions concentrative kinetic functions into a single point (s) in a compact integrated design. The question is characterized by the integration of solutions: Using the joystick motion module moves the mechanism, multiple motion module moves aggregative mechanism regulating module adaptable motion kinetic power (speed) mechanism.

Each of these solutions corresponds to a specific concept $[11,12]$. We focus on the joystick-motion 2D module.

\subsection{Profiling module motion with respect to functional and technological modularity}

In the development of construction components, modules and integrated assemblies, as is documented here, is constantly increasing requirements for operational and technological level of modularity. Under the functional modularity means the aggregation functions of individual elements of a modular design chain, which in the case of motion module is based on the systems approach. Under the technological design modularity means individual structural elements aggregated in the movement module compatible with the technological requirements of the machine system. As a rule, the level of technical parameters, requirements arising from the disposition of technology, power supply, signal and control signals via the media and module design elements, includes requirements for closure of the module Fig. 3.

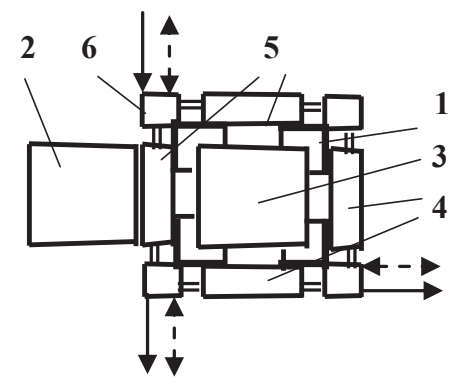

1-Reference base

2-Motion

3-transformation and distribution of elements

4-Transmission elements

5-Interconnection elements

6-Member of the collection and distribution of energy and information

$\longrightarrow$ Energy flow

$\leftarrow-\rightarrow$ Information flow

Fig. 3: Compatible motion module.
In the interface between mechanical, energy and motion control functions of the module are critical ways to connect, either through the flange /5/, clutch /4/ or through joint collection and distribution base energy supply, measurement and control signals /6/. The higher integration can be up to the unification parts base, such as a common output-connecting element (turntable, sane), the joint solution to brake and position throughout the measurement module and further integration of individual components.

From the point of view it is important to distinguish the degree of compactness of the individual components and technology Compatibility, i.e. like that module structurally, functionally capable of interfering energy, managing connections needed for final technology (service) process on the machine and what level of coverage is the solution.

\subsection{Reference solution}

The concept of 2D motion module comprises transmission and transformation mechanisms in an integrated whole. It respects the fundamental requirement to ensure the rotation, sliding and general movements, which are carried out operations to change the location, position and orientation of the object (instrument), without changing the position of the beginning of local coordinate systems. Query on motion integration in several coordinate axes is technically feasible through the module as propulsion, transmission and transformation member whose profile has the form:

\section{- parallel system,}

- integrated serial system.

The first form of a parallel kinematics, which consists of several parallel, fold a length of mechanical rods, whose length is permuted motor or automatic. Bars represent the integrated system of motion axes that are connected to the frame and the Task Force through ball or cardan joints. The main advantage of parallel kinematics is the realization of incremental movements in conformity with the common coordinate system.

In the latter case it is a nonstandard integration [12], two series-arranged movements of nodes into a module with integrated movements - Fig. 4. The essence of the solution is to transform the series into compact unit kinematics reconfiguration effects of generation and transformation of the kinetic forces. The shape of the module can be transferred 


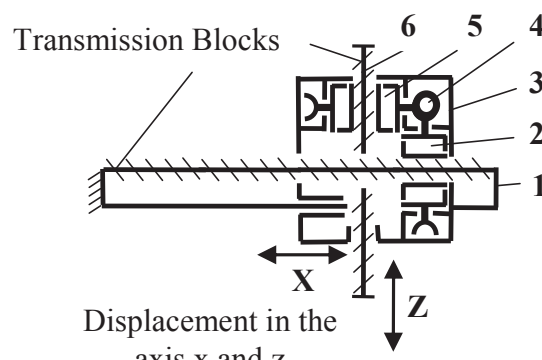
axis $\mathrm{x}$ and $\mathrm{z}$

a. Scheme of mechanism

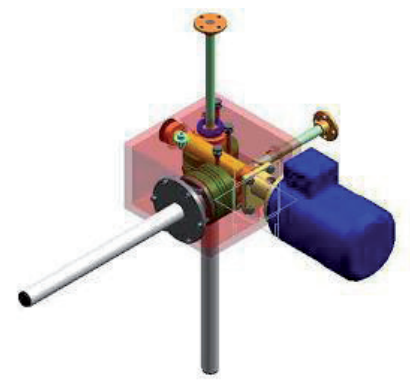

$\equiv$ Energy block

$\equiv$ Transformation and distribution block

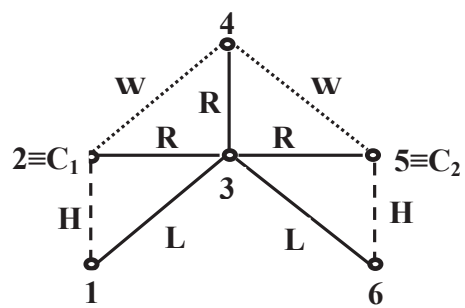

$\mathrm{R} \square$ Rotation constraints, L $\square$ Linear constraints, $\mathrm{H} \square$ Helical mechanism, W $\square$ Worm mechanism, $\mathrm{C} \square$ Clutch mechanism

b. Kinematics chain

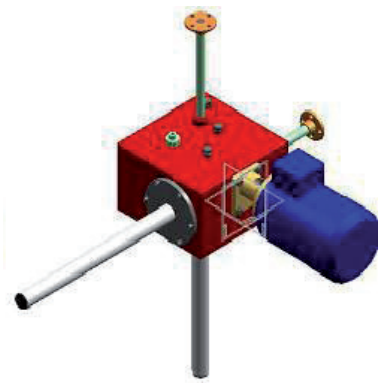

Fig. 4: 2D motion module with integrated movements.

in modifications: displacement-displacement, displacement-rotation, rotation-rotation.

Solution of Fig. 4 integrating movement (displacement in the axis $x$ and $z$ ) consists of energy, transformation, distribution and transmission-block. Unlike traditional solutions are able to pool and fork movement in automated mode as required to function in the positioning of one or two coordinate axes. Maybeitmade morecomplexand technological capacity in handling complex operations requiring special arrangements positioning and orientation, and optimal distribution of movements between the tool and object processing.

\section{Construction of Machine Chain 5.1 Principles of construction}

Introduced the concept reflected a requirement to increase and accelerate the technical-economic level of integration of technology, handling, transport and storage under multimodality. Route multimodality integration represents a new form of design and technology links functional modules. Through functional elements (nodes) capable of integration and creating confusion features a compact machine chain. Allows more functions implemented directly (the movement, positioning and orientation). This creates scope for reducing the autonomous creation of new models and designtechnological solutions. Depending on the type of grouping, layout and construction-technological links we can develop machine systems to new and more integrated level, i.e. in the form:

- of the subject module (adjustable and retractable rotary table for the cultivation of the five parties),

- the basic model (MS), as applicable substitute machine (for keeping the arm member working in conjunction with the positioning tables for easy work activities),

- complex (discrete multi-system) model (CMS) as a multimodal production / handling systems (integrated kinematics for the implementation of more demanding job tasks associated with the performance and handling technology tasks),

- logistics (an integrated multi-system) model (LMS) as a special (professional) machine systems (multiple integrated kinematics for the implementation of more demanding job tasks associated with the object profiling more axes) - Fig. 5.

Determining fact for the development of machine systems is that design and technological ties are extracted from machine-base (reference module), and are distinguished by hierarchical level.

The core of the solution of logistic models immediate concentration of functions and activities 
directly between tool and subject module system. This requires continuous addressing the structural and technological ties of tool of the subject modular system in order to mutually integrate their physical activities. Today these solutions are presented as logistical and are included under the new science intralogistics, whose importance lies in the detection of new principles of internal and external integration (e.g.: mechanical development, energy and information links) modules, assemblies and systems. Logistic structure of the machinery of the system (LMS) is symbolically expressed by the relation (1).

An example of realization of logistic structure of machine system is Figure 5 using multiple integration of re-configurable movement modules. A specific of solution is that multiple integrated kinematics $X, Z$ created by two parallel pairs of jointed arms. The first endings of the two arms are connected by platforms providing on one hand the movement of tool, and movement of processed object on the other hand. The other endings of the pair of arms are provided with multi-function joint, which in turn ensures distribution of physical force in the dependent and independent modes of action and subject-tool branches.

Special (professional) machine system - Fig. 5 is another way to improve the techno-economic level of the integration of multiple modules reconfiguration motion. The specific solution is that multiple integrated kinematics $X, Z$ are arranged in two parallel pairs of jointed arms. The first pair of arm ends are connected platforms providing the first movement of the instrument, and secondly, the movement of object processing. The second ends of pairs of arms are provided with multi-functional joint, which in turn will ensure distribution of the kinetic power in the dependent and independent modes of action and the curricular tool line.

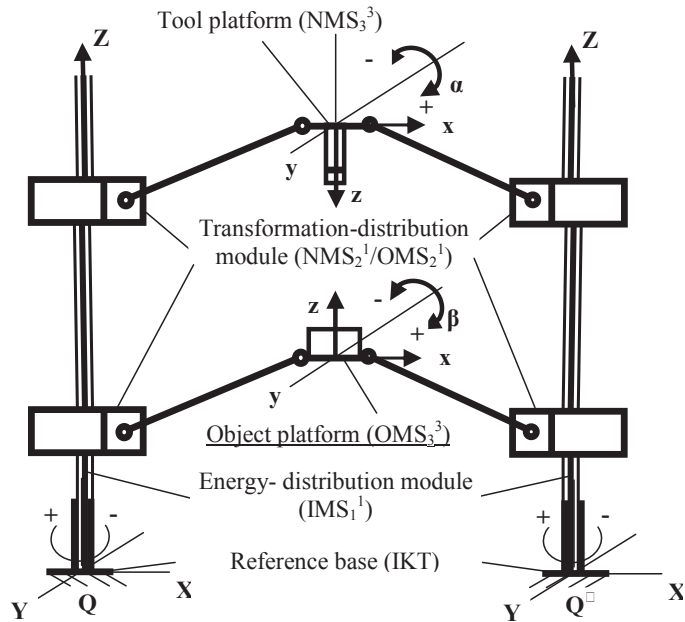

Fig. 5: Special machine system.

Multiple integrated kinematics made partial movements in various stages of activity. Movements are being diverted away from the common linear propulsion system, through which the tool path moving in the coordinates $X, Z$ and A (typically, this may be milling headstock) rebuilt to object tied to a fixed (floating) base respectively contrary. Otherwise, operation mode tool platform is meant to frame a working unit becomes subject platform (positioning table) moving coordinates $X, Z$ and $B$. This integration results in the so-called multiple series-parallel kinematics represents non-standard reconfigurable machine system. The solution is designed to implement the demanding workloads (transmission and operational procedures for handling and profiling facility) requiring special arrangements positioning and orientation without the need for further integrated devices. Compared with individual modules using single functional programmed multi occupational manufacturing module with the wider effects of variability (range of technology, quality, and greater time effect).

$$
L M S=I K T \stackrel{k, t, v}{\longleftrightarrow}\left\{\bigcup_{1}^{2}\left\{I M S_{1}^{1}\right\} \stackrel{k, z}{\longleftrightarrow}\left\{\left\{\int_{1}^{2}\left\{\left\{N M S_{2}^{1}\right\}\right\} \cap\left\{N M S_{3}^{3}\right\}\right\} ;\left\{\left\{\bigcup_{1}^{2}\left\{O M S_{2}^{1}\right\}\right\} \cap\left\{O M S_{3}^{3}\right\}\right\}\right\}\right\}
$$

\section{when:}

IKT - multifunctional integrated design and technology base (reference module), $I M S_{1}^{1}$ energy- distribution module, $N M S_{2}^{1} / O M S_{2}^{1}$ transformation-distribution module, $N M S_{3}^{3}$ - tool platform, $O M S_{3}^{3}$ - object platform, $\stackrel{k, t, v}{\longleftrightarrow}$ - symbolic expression of design and technological capabilities and linkages variations, $\stackrel{k, z}{\longleftrightarrow}$ symbolic expression of structural links and pooling of skills / substitution motion activities in the machine system. 


\subsection{Application Characters}

Modular reconfigurable machinery and systems consisting of individual modules can be flexibly combined. Moreover, can integrate additional features, such as adding motion-individual modules vary operational and working opportunities for machine system. The solution is based on structural and technological elements and variable modules capable of reducing and mutual transformation of the members of the construction chain, which allows developing multifunctionality, adaptability and reconfigurability for direct and reversible operation. The result is a new configuration options handling, manufacturing and supporting technology, it's lean and create inventive combinations of new and higher technical and economic effects. The results of the work will be used to expand or modernize as its manufacturing base and broad impact on production applications based on lean machine systems constituting of new positioning elements in machining, welding and assembly.

The development of machine systems is closely related to innovation and modernization program, business services (technology, handling, transport and storage). They apply to: multimodality solving machine (production, handling, transport, storage) systems, peripherals expansion of production machinery (manufacture of shape and space for complex products), the modernization of the older generation and handling technology, retrofit motion modules with extending the current work than in new axis technology, operational security engineering and production-type contracts, representing a system integrator working with higher demands on flexibility and broad spectrum.

Typical reconfiguratible machinery product group of modules and systems are the subject of the tooling and production machines, working groups for handling and transposition of instruments / objects are particularly useful for building and working positioning modules required to ensure that current movements in a particular place at a high manoeuvrability.

A preliminary estimate using this technique in industrial practice is most likely the production of components, integration of product design (assembly, disassembly) and the integration of products for the market (packaging, palletizing). Particular category of applications is profiling, streamlining and creating inventive solutions supporting the production and handling technology in engineering.

\section{Conclusions}

Modular reconfigurable machinery and systems consisting of individual modules can be flexibly combined. Moreover, can integrate additional features, such as adding motion-individual modules vary operational and working opportunities for machine system. The solution is based on structural and technological elements and variable modules capable of reducing and mutual transformation of the members of the construction chain, which allows developing multifunctionality, adaptability and reconfigurability for direct and reversible operation. The result is a new configuration options handling, manufacturing and supporting technology, it's lean and create inventive combinations of new and higher technical and economic effects. The results of the work will be used to expand or modernize as its manufacturing base and broad impact on production applications based on lean machine systems based on new positioning elements in machining, welding and assembly

\section{Acknowledgments}

This work has been supported by the Slovak Grant Agency VEGA contract Nb. 1/0124/15 "Research and development of advanced methods for virtual prototyping of production machinery".

\section{References}

[1] Beard, T.: Feature - New Turning Centre Design Aims at Maximum Turning and Milling Rates. Mazak's MMS OnlineTM, 2005, http://www.mmsonline. com/articles/ 070406. html.

[2] COP, V.: Development projects of production techniques and technologies. SPINEA s r.o., Presov, 2005

[3] Koepfer, C.: Rapid Traverse - One Stop Milling, Drilling, Turning and Grinding. Mazak's MMS OnlineTM, 2005, http:// www.mmsonline. com/articles/ 1201rt2.html.

[4] Koren, Y., Ulson, G.: Vision, Principles and Impact of Reconfigurable Manufacturing Systems. Powertrain International, 2002, pp. 14-21.

[5] Kusiak, A.: Design for Agile Assembly: An Operational Perspective, International Journal of Production Research, Vol.35, No.1, 1997, pp.157-178.

[6] LIPSON, H., POLLACK, J.B.: Automatic design and manufacture of robotics life forms. Nature 406, pp. 974-978.

[7] Mehrabi, M., Ulsoy, A. G., Koren, Y.: Reconfigurable Manufacturing Systems: Key to Future Manufacturing, J. of Intel- 
ligent Manufacturing, Vol. 11, № 4, 2000, pp. 403-419.

[8] VALENCIK, S.: The principles and acceptable solutions of modular structures handling device. Strojarstvo 12/2000, MEDIA-ST s.r.o. Bratislava, 2000, s. 38-39. (In Slovak)

[9] VALENCIK, S.: Design and application of multifunction hydraulic drive system. AUTOMA 1/2004, FCC PUBLIC, Praha, 2004, s. 12-14. (In Slovak)

[10] VALENCIK, S.: Solution motion integration of robotic technique modules. ATP Journal plus 2 2/2006, Bratislava, 2006, s. 96-99. (In Slovak)

[11] VALENCIK, S.: Specification architecture and development of reconfigurable machine system. In: MM Science Journal. No. 1 (2011), p. 232-235. (In English)

[12] VALENCIK, S.: Development and application of 2D dynamic module. In: MM Science Journal. No. 1 (2012), p. 294-297. (In English)

[13] Shaw, R:: Better Production - Manufacturer Takes Innovative Approach to Advanced Manufacturing Processes, Mazak's MMS OnlineTM, 2005, http://www.mmsonline. com/articles/ 0500bp4.html.

[14] Sheridan, J. H.: Agile Manufacturing: Step Beyond Lean Manufacturing, Industry Week, 1993, pp. 30-46.

\section{Biographical notes}

Štefan Valenčík, doc., Ing., CSc., (born in1953) is associated professor at he Technical University of Košice, Faculty of Mechanical Engineering, Dept. of Production Techniques. He received M.S. degree in mechanical engineering from Technical University of Košice, Slovakia in 1977, and obtained his degree as Candidatus Scientiae in 1985, associate professor (2001). His research profile consists of Automation, Robotics, Automated Manufacturing, Production machinery, Designing and Construction. He is an author of one monograph, author/co-author of five textbooks, his five papers were registered in Web of Science and SCOPUS. 